\title{
Gadolinium-Based Contrast Agents Hypersensitivity: A Case Series
}

This article was published in the following Dove Press journal:

Journal of Asthma and Allergy

\author{
Eleonora Nucera' \\ Sara Urbani $\mathbb{D}^{\prime}$ \\ Alessandro Buonomo' \\ Tiziana De Pasquale ${ }^{2}$ \\ Angela Rizzi' \\ Arianna Aruanno' \\ Stefano Pucci ${ }^{2}$ \\ 'Allergy Unit, Fondazione Policlinico \\ Universitario A. Gemelli IRCCS, Rome, \\ Italy; ${ }^{2}$ Civitanova Marche Hospital, \\ Civitanova Marche, Italy
}

\begin{abstract}
Gadolinium-based contrast agents (GBCAs) are considered to be safe, although sometimes patients report a hypersensitivity reaction when undergoing magnetic resonance imaging (MRI). The mechanisms of these reactions and of the sensitization to GBCAs are still largely unknown. We describe four cases of patients who experienced immediate adverse reactions to GBCAs with a demonstrated cutaneous hypersensitivity suggesting an IgEmediated mechanism.
\end{abstract}

Keywords: gadolinium-based contrast agents, hypersensitivity, IgE-mediated reactions

\section{Introduction}

Gadolinium-based contrast agents (GBCAs) have been used for magnetic resonance imaging (MRI) since the 1980s, when the first GBCA, gadopentetate dimeglumine, became available for clinical use. Since then, GBCAs have been used annually in approximately 30 million procedures, with more than 300 million procedures performed to date worldwide. ${ }^{1}$ The GBCAs are classified on the basis of their molecular structure and their net charge into linear ionic (gadopentetate dimeglumine, gadobenate dimeglumine, gadoxetate disodium and gadofoveset trisodium), linear non-ionic (gadodiamide and gadoversetamide), macrocyclic ionic (gadoterate meglumine) and macrocyclic non-ionic (gadobutrol and gadoteridol). Despite their widespread global use, the overall rate of patients who experienced immediate hypersensitivity reactions (HR) was 9.2 per 10,000 administrations. ${ }^{2}$ Risk factors for GBCAs hypersensitivity include history of allergies and asthma, a previous reaction to a GBCA, female gender, repeated exposure to GBCAs and systemic mastocytosis. $^{3}$ The clinical manifestations of GBCAs hypersensitivity are extremely variable, from skin symptoms to fatal anaphylaxis; however, urticaria is the most common clinical presentation, occurring in $91 \%$ of hypersensitivity reactions. ${ }^{4}$ The mechanism of HRs to GBCAs may be immunological, either IgE- or non IgEmediated. Other immunological mechanisms may be due to the following pathways: (1) direct membrane effect on mast cells and/or basophils; (2) complement activation by $\mathrm{IgG}$ antigen complexes; (3) bradykinin generation. ${ }^{3}$

In the literature there are few reports on IgE-mediated reactions and no conclusions on cross-reactivity can yet be drawn.

\section{Case Series}

We report four cases of GBCAs hypersensitivity documented by positive immediate-type skin test results (Table 1). All presented patients were female with
Allergy Unit, Fondazione Policlinico

Universitario A. Gemelli IRCCS, Largo

F. Vito, I, Rome, 00168 , Italy

Tel +393394475931

Fax +0630156999

Email eleonora.nucera@policlinicogemelli.it 


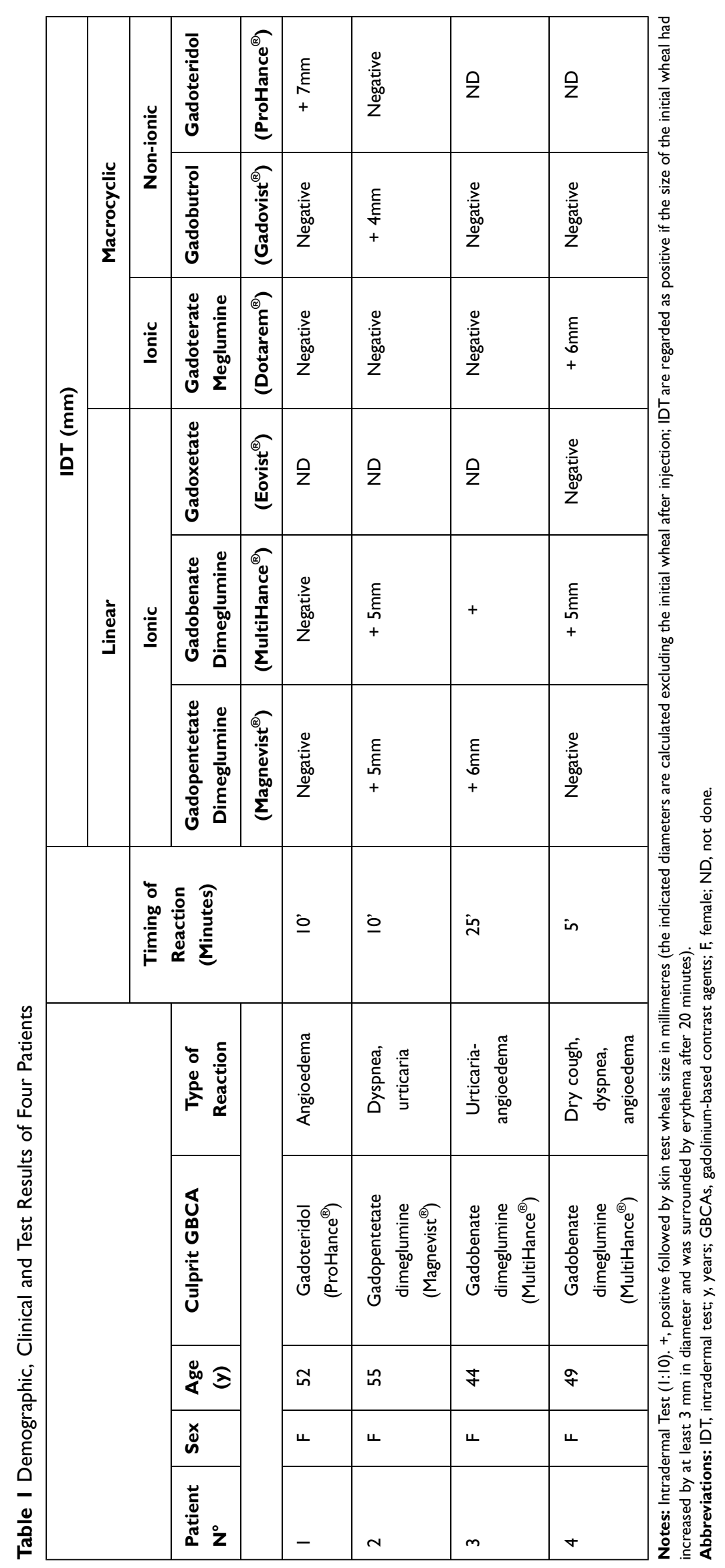




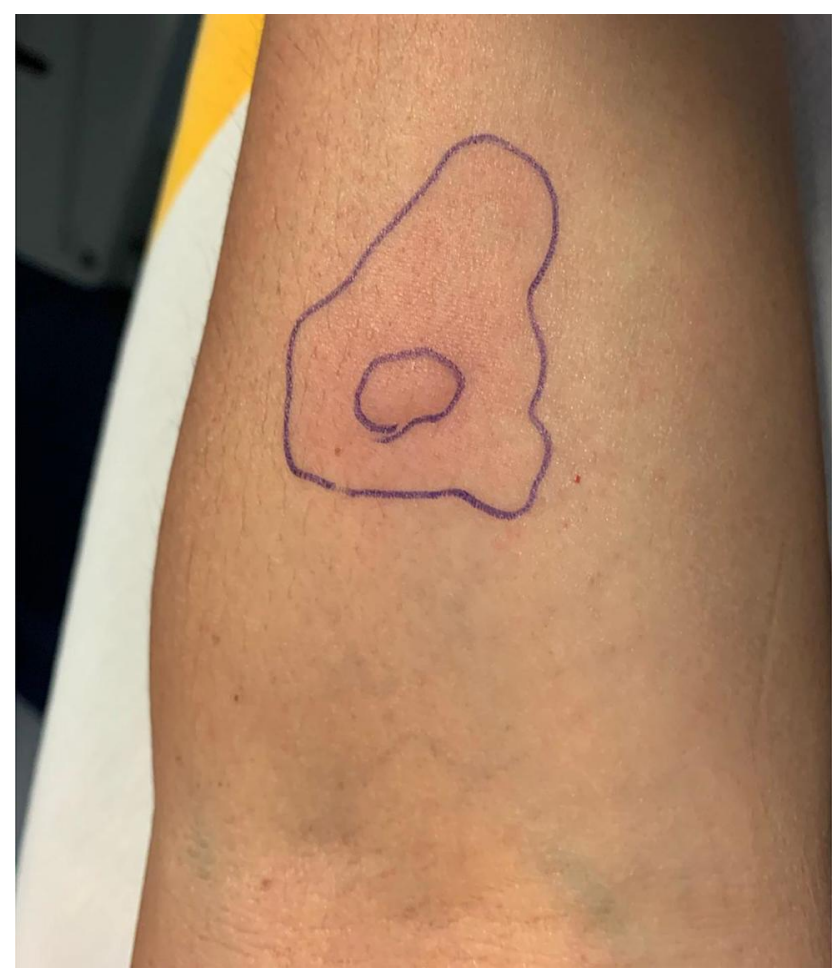

Figure I Case I gadoteridol IDT result with main size of wheal of $9 \mathrm{~mm}$ and flare of $4 \mathrm{~cm}$ starting from $2 \mathrm{~mm}$ after GBCA injection (histamine wheal of $4 \mathrm{~mm}$ and negative control wheal of $\mathrm{I} \mathrm{mm}$ ).

ages ranging between 44 and 59 years who contacted our institutes for an allergological evaluation from September 2018 to January 2020. Two patients had a history of allergy comorbidity (allergic rhinitis and adverse reaction to other drugs) but none had an asthma diagnosis. In three of our cases the involved agent was gadobenate dimeglumine, in case 1 the reaction was caused by gadoteridol. In all cases, the hypersensitivity reactions had an acute presentation, appearing within the first hour after administration, characterized by cutaneous (urticaria, angioedema, pruritus) or respiratory (dyspnea, dry cough) symptoms; the symptoms had anaphylactic features in only one case. In one case, the adverse reaction occurred after repeated exposure to GBCAs. Skin tests were performed on the volar forearm in accordance with EAACI recommendations ${ }^{5}$ and a diameter greater than $3 \mathrm{~mm}$ was considered a positive response for an immediate reading at 15 minutes. The intradermal test (IDT) was regarded as positive if the size of the initial wheal had increased by at least $3 \mathrm{~mm}$ in diameter and was surrounded by erythema after 20 minutes. Histamine and saline solution were used, respectively, as positive and negative controls. For each presented patient, firstly, we performed a skin prick test (SPT) with undiluted GBCAs, and, if negative, IDT using a dilution of $1: 10 .^{5}$ Within 3 months from the reactions, all patients were tested with the culprit agent and with alternative molecules to assess crossreactivity and to provide a safe alternative molecule. ${ }^{5}$ In three cases, we found positive skin test reactions to both linear and macrocyclic GBCAs. In case 1 (Figure 1), we also performed a basophils activation test (BAT) with a negative result and the basal serum tryptase level was normal at 4 weeks after the reaction, excluding a systemic mastocytosis disease. In three cases, we found a skin reaction cross-reactivity between linear and macrocyclic GBCAs.

\section{Discussion}

This case series report showed an immediate reaction to GBCAs documented by skin tests. The exact pathophysiologic mechanism of GBCAs hypersensitivity is largely unknown. Some hypotheses support the involvement of activation of mast or basophils cells or a direct histamine release. ${ }^{6,7}$ However, the positivity of ID tests gives a strong argument for IgE-mediated reactions. Indirect evidence for a serum $\operatorname{IgE}$ pathogenic role was provided by our research group through a Prausnitz-Kustner test. ${ }^{8}$ As Jung et al. previously indicated, ${ }^{4}$ in our experience, gadobenate dimeglumine was the more frequent agent implicated in the hypersensitivity reactions. Differently, in a retrospective analysis of patients investigated by Mankouri et al. ${ }^{9}$ gadoteric acid was the most frequently GBCA reported by patients as the cause of a reaction, followed by gadobenic acid. Two of the three patients who had a reaction with a linear GBCA showed a cross-reactivity also for a macrocyclic agent having the same net charge. Cross-reactivity in skin tests has recently been demonstrated in one third of allergic patients. ${ }^{9}$ However, some previous reports showed no crossreactivity between GBCAs ${ }^{10,11}$ while others showed crossreactivity between gadobutrol and gadoteridol ${ }^{12}$ and between gadobutrol and several both linear and macrocyclic agents. ${ }^{9,13}$ These findings cannot allow us to draw any conclusion on cross-reactivity and further studies are needed to clarify this mechanism. In our experience, only the case 2 showed the reaction after repeated exposures to GBCAs, while the other cases presented symptoms during their first exposure. Reaction after first exposure to a drug has already been described by Hasdenteufel et al. ${ }^{14}$ and could be due to work exposure (in metallurgical plants, magnet manufacturers, fluorescent lamps, or television sets), to exposure to GBCAs in drinking water ${ }^{15}$ or to particular characteristics of 
these drugs. Based on high reported negative predictive value, ${ }^{9,14}$ in previous reactors skin testing seems to be a useful tool in selection of a safe product for their future MRIs. For these reasons, although none of our patients have undergone further MRIs, we advised them to use negative skin test for GBCAs for the future. Our findings support the importance of accurate allergy assessment, principally skin tests, to document the drug's involvement. Indeed, in the case of a reaction to a GBCA, the recognition of allergic features as well as performing skin testing are important to determine the immunological etiology of the reaction and to find a safe alternative.

\section{Statements of Ethics}

All subjects have given their written informed consent and they have given their consent to publish the case details in this paper.

This work obtained institutional approval by Fondazione Policlinico Universitario A. Gemelli of Rome according with the Declaration of Helsinki.

\section{Acknowledgments}

The authors are responsible for the content and the writing of this paper.

\section{Author Contributions}

All authors contributed to data analysis, drafting or revising the article, have agreed on the journal to which the article will be submitted, gave final approval of the version to be published, and agree to be accountable for all aspects of the work.

\section{Funding}

The authors declare that no funding was received for the present work.

\section{Disclosure}

The authors report no conflicts of interest in this work.

\section{References}

1. Lohrke J, Frenzel T, Endrikat J, et al. 25 Years of contrast-enhanced MRI: developments, current challenges and future perspectives. $A d v$ Ther. 2016;33(1):1-28.

2. Behzadi AH, Zhao Y, Farooq Z, et al. Immediate allergic reactions to gadolinium-based contrast agents: a systematic review and meta-analysis. Radiology. 2018;2(2):471-482. doi:10.1148/radiol.2017162740

3. Costello JR, Kalb B, Martin DR. Incidence and risk factors for Gadolinium-based contrast agent immediate reactions. Top Magn Reson Imaging. 2016;25(6):257-263. doi:10.1097/RMR.0000000000000109

4. Jung JW, Kang HR, Kim MH, et al. Immediate hypersensitivity reaction to Gadolinium-based MR contrast media. Radiology. 2012;264(2):414-422. doi:10.1148/radiol.12112025

5. Brockow K, Garvey LH, Aberer W, et al. Skin test concentrations for systemically administered drugs - an ENDA/EAACI drug allergy interest group position paper. Allergy. 2013;68(6):702-712. doi:10.1111/all.12142

6. Javaloyes G, Goikoetxea MJ, Sanz ML, et al. Basophil activation test in the diagnosis of gadobutrol anaphylaxis. Ann Allergy Asthma Immunol. 2012;108(4):280-287. doi:10.1016/j.anai.2012.02.003

7. Kun T, Jakubowski L. Influence of MRI contrast media on histamine release from mast cells. Pol $J$ Radiol. 2012;77(3):19-24. doi:10.12659/PJR. 883370

8. Schiavino D, Murzilli F, Del Ninno M, et al. Demonstration of an IgE-mediated immunological pathogenesis of a severe adverse reaction to gadopentetate dimeglumine. J Investig Allergol Clin Immunol. 2003;13(2):140-142.

9. Mankouri F, Amélie G, Witchaya S, et al. Hypersensitivity to gadolinium-based contrast agents: a single-center retrospective analysis over 7 years. J Allergy Clin Immunol Pract. 2020;S2213-2198(20)31244-7. doi:10.1016/j.jaip.2020.11.023

10. Sellaturay P, Nasser S, Ewan P. Skin testing in gadolinium allergy: 2 case reports. J Allergy Clin Immunol Pract. 2018;6(1):262-264.e1. doi:10.1016/j.jaip.2017.04.040

11. Rodriguez-Nava G, Kesler AM, Carrillo-Martin I, Gonzalez-Estrada A. Gadolinium-induced anaphylaxis with positive skin test results. Ann Allergy Asthma Immunol. 2019;122(6):654-655. doi:10.1016/j. anai.2019.03.022

12. Moreno Escobosa MC, Cruz Granados S. Paramagnetic contrast media: hypersensitivity and cross-reactivity. $J$ Investig Allergol Clin Immunol. 2018;28(1):60-62. doi:10.18176/jiaci.0210

13. Böhm I. Iodinated X-ray contrast media in aquatic environment in general and in drinking water in particular: a possible source for the primary sensitization of patients. Chemosphere. 2018;194:28-29. doi:10.1016/j.chemosphere.2017.11.154

14. Hasdenteufel F, Luyasu S, Renaudin JM, et al. Anaphylactic shock after first exposure to gadoterate meglumine: two case reports documented by positive allergy assessment. $J$ Allergy Clin Immunol. 2008;121(2):527-528. doi:10.1016/j.jaci.2007.08.027

15. Chiriac AM, Audurier Y, Bousquet PJ, Demoly P. Clinical value of negative skin tests to gadolinium contrast agents. Allergy. 2011;66 (11):1504-1506. doi:10.1111/j.1398-9995.2011.02690.x
Journal of Asthma and Allergy

\section{Publish your work in this journal}

The Journal of Asthma and Allergy is an international, peer-reviewed open-access journal publishing original research, reports, editorials and commentaries on the following topics: Asthma; Pulmonary physiology; Asthma related clinical health; Clinical immunology and the immunological basis of disease; Pharmacological interventions and new therapies. The manuscript management system is completely online and includes a very quick and fair peer-review system, which is all easy to use. Visit http://www.dovepress.com/testimonials.php to read real quotes from published authors. 